\title{
Aspectos da vacinação contra hepatite $B$ em idosos, no município de Salvador (BA), de 2004 a 2018: um estudo descritivo a partir do Sistema Eletrônico do Departamento de Informática do SUS (DATASUS)
}

\author{
Aspects of hepatitis B vaccination in the elderly of Salvador (BA) from 2004 to 2019: a \\ descriptive study based on the Electronic System of the SUS Department of Informatics
} (DATASUS)

Viviane da Conceição Davino de Assis ${ }^{1}$, Denise Carneiro Lemaire ${ }^{2 *}$

\begin{abstract}
${ }^{1}$ Nutricionista. Mestranda do Programa de Pós-graduação em Processos Interativos de Órgãos e Sistemas, da Universidade Federal da Bahia; ${ }^{2}$ Professora plena da Universidade do Estado da Bahia; Biomédica. Doutora em Imunologia.
\end{abstract}

\begin{abstract}
Resumo
Introdução: a hepatite B é um grave problema de saúde pública no país e no mundo. Nos últimos dez anos, houve aumento significativo do número de casos notificados em idosos. Embora seja uma doença imunoprevinível, há poucos dados na literatura sobre a situação vacinal deste grupo populacional. Objetivo: este estudo visou investigar e descrever dados sobre a vacinação para hepatite B no município de Salvador, Bahia, registrados no Sistema eletrônico do Departamento de Informática do SUS (DATASUS) do Ministério da Saúde. Materiais e métodos: Trata-se de estudo descritivo, a partir de dados secundários disponíveis no Sistema de Informações do Programa Nacional de Imunizações e Sistema de Informação de Agravos de Notificação, no período 2004 a 2018, referentes ao município de Salvador, Bahia. Resultados: foram notificados 120 casos de hepatite B em idosos, no período estudado. A principal forma de contágio foi pela via sexual. Os maiores números de doses aplicadas da vacina foram observados nos anos 2017 e 2018, principalmente da primeira dose. Este aumento está, provavelmente, associado ao fato de que, em 2016, esta vacina passou a ser disponibilizada de forma universal, independentemente de idade ou condições de vulnerabilidade. Contudo, a proporção da população idosa residente no município de Salvador vacinada foi muito baixa $(<1 \%$ ao ano). Conclusão: este estudo revelou baixa cobertura vacinal da população de idosos de Salvador. Estes dados reforçam a importância de estratégias no planejamento de programas de atenção à saúde do idoso que incentivem maior adesão à vacinação completa contra hepatite $B$.
\end{abstract}

Palavras-chave: Vacinação. Hepatite B. Idosos.

\begin{abstract}
Introduction: hepatitis B is a worldwide public health problem. The occurrence of this disease in the elderly has increased significantly over the past 10 years. Although it is a vaccine-preventable disease, information about vaccination status in this population group is scarce. Objective: this paper aims to investigate and describe hepatitis B vaccination data in the elderly in Salvador, Bahia. Methods: it is a descriptive study, which collected data available at the Information System of the National Immunization Program, and at the Information System of Notification of Diseases websites. The years included in this research were from 2004 to 2018, and the resident population was from Salvador, Bahia, Brazil. Results: in the study period, 120 cases of hepatitis B were reported in the elderly in Salvador. The main route of infection was through sexual intercourse. The highest numbers of hepatitis $B$ vaccine injections are observed in 2017 and 2018, especially the first shot (out of three shots). This increase may be associated with the government measure that ensured hepatitis $B$ vaccination for every Brazilian citizen, regardless his/her age or vulnerability since 2016. However, the proportion of the vaccinated elderly population living in Salvador is still very low ( $<1 \%$ per year). Conclusion: this study shows low hepatitis $B$ vaccination coverage of the elderly living in Salvador, Bahia. These data reinforce the importance of strategies that prompt elderly health care programs to encourage greater adherence to hepatitis B complete vaccination by older population.
\end{abstract}

Keywords: Vaccination. Hepatitis B. Elderly.

\section{INTRODUÇÃO}

A hepatite $B$, um grave problema de saúde pública no Brasil e no mundo, é uma doença de notificação compul-

Correspondente/Corresponding: *Denise Carneiro Lemaire Departamento de Ciências da Vida. Universidade do Estado da Bahia - End.: Rua Silveira Martins, s/n, Cabula, Salvador-BA. CEP: 40301 - 110 - Tel: (71) 98737-2567 -E-mail: dlemaire@uneb.br sória desde $1999^{1,2}$. De acordo com o Boletim Epidemiológico de Hepatites Virais, do Ministério da Saúde, 233.027 casos de hepatite $B$ foram confirmados, no período de 1999 a $2018^{2}$. Este dado é ainda mais preocupante pelo fato de que muitos indivíduos infectados pelo vírus da hepatite $B$ (HBV) evoluem para a forma crônica da doença, podendo desenvolver cirrose, insuficiência hepática e carcinoma hepatocelular ${ }^{3}$.

Nos últimos dez anos, as taxas de incidência 
da hepatite B no Brasil pouco variaram e apresentaram uma tendência de leve queda, a partir do ano de 2014. Entretanto, no período entre 2008 e 2018 , foi observado aumento significativo das taxas de incidência da doença na população com idade maior ou igual a 60 anos². De $^{2}$ acordo com o Instituto Brasileiro de Geografia e Estatística (IBGE), projeções baseadas nos últimos Censos de 2000 e 2010 mostraram que, no Brasil, também tem sido observado o fenômeno de envelhecimento crescente da população. Essa elevação pode estar relacionada, em parte, ao aumento da expectativa de vida e a redução da taxa de fecundidade ocorrida nos últimos anos ${ }^{4}$.

$\mathrm{O}$ aumento do número de idosos foi acompanhado pelo prolongamento de sua vida sexual, resultante do desenvolvimento de novas terapias de tratamento da impotência masculina, embora associadas a práticas sexuais inseguras ${ }^{5}$. A via sexual é uma importante forma de transmissão do HBV sendo, inclusive, considerada pelo Ministério da Saúde, uma infecção sexualmente transmissível $^{6}$. É importante destacar que idosos infectados pelo HBV têm maior risco de evoluir para a forma crônica e de desenvolver complicações?

A vacinação é a forma mais segura e eficaz de prevenção da infecção pelo HBV em todas as faixas etárias ${ }^{8}$. Para que o indivíduo seja considerado imunizado é necessária a aplicação das três doses da vacina, com intervalos de 30 dias da primeira para a segunda dose e 180 dias da primeira para a terceira dose. ${ }^{6}$ Assim, é fundamental o desenvolvimento de políticas sociais e de saúde que visem incentivar o aumento da cobertura vacinal contra a hepatite $B$ na população idosa. $O$ calendário de vacinação do adulto e do idoso foi instituído no Programa Nacional de Imunizações (PNI), do Ministério da Saúde, com a publicação da Portaria $n^{\circ} 597$, de 8 de abril de 2004. É importante destacar que essa vacina foi ofertada, inicialmente, para a população da Amazônia Ocidental e passou a ser aplicada em crianças com menos de um ano em 1998. A partir de 2010, a oferta desse imunobiológico foi progressivamente ampliada para outras faixas etárias ${ }^{9}$. Em 2016, foi disponibilizada, de forma universal, para população brasileira, independentemente de idade ou condições de vulnerabilidade (Nota Informativa no 149/2015, do Ministério da Saúde $)^{10}$. Apesar de a vacina atualmente estar disponível, gratuitamente, nas Unidades Básicas de Saúde, ser segura e eficaz, relatório divulgado pela Coordenadoria do Programa Nacional de Imunizações (PNI) revelou baixa cobertura vacinal, no território brasileiro, em idosos no ano de $2018^{11}$. A baixa adesão à vacinação contra a hepatite $B$, nesse grupo, contribui significativamente para o aumento do risco de adoecimento dos idosos, além de ter impacto na sua qualidade de vida e, também, nos gastos em serviços de saúde pública.

No estado da Bahia, no período de 1999 a 2018, foram notificados mais de 7 mil casos de hepatite $B$ na população ${ }^{2}$. Apesar das evidências que mostram aumento da incidência da doença e maior risco de complicações em idosos, poucos estudos foram desenvolvidos sobre a cobertura vacinal da hepatite B nessa faixa etária, no município de Salvador.

Assim, o objetivo deste estudo é investigar e descrever dados sobre a vacinação para hepatite $B$ no município de Salvador, Bahia, registrados no Sistema Eletrônico do Departamento de Informática do SUS (DATASUS), do Ministério da Saúde.

\section{METODOLOGIA}

Trata-se de um estudo descritivo exploratório, a partir de dados secundários provenientes do DATASUS, disponibilizados pelo Sistema de Informações de Saúde (TABNET) do Ministério da Saúde.

Os dados relativos à vacinação para hepatite $B$ em idosos, na Bahia, no período de 2004 a 2018, foram obtidos pelo acesso ao Sistema de Informações do Programa Nacional de Imunizações (SI-PNI) ${ }^{12}$. Este período foi escolhido para avaliar a evolução da proporção de idosos vacinados, a partir do ano em que foram instituídos os calendários de vacinação por ciclo de vida, incluindo o do adulto e do idoso, no PNI. As seguintes variáveis foram selecionadas: ano - período de janeiro de 2004 a dezembro de 2018; sexo - ambos os sexos; faixa etária - igual ou superior a 60 anos; e número de doses aplicadas da vacina para hepatite $B-a$ fim de averiguar proporção de idosos com esquema vacinal completo, dentre os vacinados.

Os dados referentes ao número de casos confirmados de hepatite $B$ em idosos e suas principais fontes ou mecanismos de transmissão foram obtidos pelo acesso ao Sistema de Informação de Agravos de Notificação $(\text { SINAN })^{13}$.

A proporção de idosos residentes em Salvador, Bahia, vacinados contra a hepatite $B$ foi obtida pelo cálculo da razão entre o número doses da vacina aplicada no período de 2004 a 2018, disponível no SI-PNI, e a população estimada no município, nesse mesmo período (IBGE) ${ }^{14}$.

Os dados obtidos do TABNET/DATASUS foram inseridos em um banco de dados, com uso do software Microsoft ${ }^{\circledR}$ Excel $^{\circledR}$ versão 2017 (Microsoft Corporation da Impressa Systems, Santa Rosa, Califórnia), programa que também foi usado para a análise dos dados e apresentação e elaboração das tabelas.

Pelo fato de o estudo ter utilizado banco de dados de domínio público, não houve necessidade da submissão do estudo ao Comitê de Ética em Pesquisa.

\section{RESULTADOS}

De acordo com o Sistema de Notificação de Agravos e de Notificação da Saúde (SINAN), nos anos 2004 a 2018 foram notificados e confirmados 5.379 casos de hepatite, causados pelo HBV no estado da Bahia. No município de Salvador, foram confirmados 1.568 casos, sendo 120 referentes a idosos, no mesmo período, conforme apresentado na Tabela 1. 
Tabela 1 - Registros de casos notificados e confirmados da hepatite B na população idosa em Salvador, Bahia, no período de 2004 a 2018.

\begin{tabular}{crrrrr}
\hline \multirow{2}{*}{$\begin{array}{c}\text { Ano Diagnósticos/ } \\
\text { sintomas }\end{array}$} & \multicolumn{5}{c}{ Faixa etária (anos) } \\
\cline { 2 - 5 } & $\mathbf{6 0 - 6 4}$ & $\mathbf{6 5 - 6 9}$ & $\mathbf{7 0 - 7 9}$ & $\mathbf{8 0}$ e + & \\
\hline 2004 & 1 & - & - & - & 1 \\
2007 & 2 & 1 & 2 & - & 5 \\
2008 & 2 & 1 & - & - & 3 \\
2009 & 1 & - & 1 & - & 2 \\
2010 & 3 & 2 & 2 & - & 7 \\
2011 & 2 & - & - & - & 2 \\
2012 & 4 & 1 & 2 & 2 & 9 \\
2013 & 7 & 3 & 6 & - & 16 \\
2014 & 2 & 2 & 1 & 1 & 6 \\
2015 & 11 & 2 & 2 & 1 & 16 \\
2016 & 8 & 7 & 2 & 2 & 19 \\
2017 & 8 & 4 & 2 & 1 & 15 \\
2018 & 8 & 4 & 6 & 1 & 19 \\
Total & 60 & 28 & 26 & 8 & 120 \\
\hline
\end{tabular}

Fonte: Ministério da Saúde/SVS - Sistema de Informação de Agravos de Notificação

Conforme com os dados mostrados na Tabela 1, a faixa etária com maior número de casos confirmados de hepatite $B$ foi a de 60 a 64 anos (60; 41,18\%), especialmente a partir de 2015.

Os dados relativos a prováveis fontes ou mecanismos de infecção pelo HBV, em Salvador, nessa faixa etária, estão mostrados na Tabela 2. Os dados sugerem que a via sexual é a principal fonte de contaminação pelo HBV em indivíduos, em particular na faixa etária referida. Essas evidências reforçam a relevância da conscientização desse grupo a respeito dos riscos da prática de atividade sexual insegura como importante via de transmissão da hepatite B.

Tabela 2 - Distribuição dos casos de idosos infectados pelo HBV em Salvador, Bahia, no período de 2004 a 2018 de acordo com a fonte ou mecanismo de infeç̧ão.

\begin{tabular}{lrrrrrr}
\hline \multirow{2}{*}{$\begin{array}{c}\text { Fonte/Mecanismo } \\
\text { de Infecção }\end{array}$} & \multicolumn{5}{c}{ Faixa etária (anos) } & Total \\
\cline { 2 - 5 } & $60-64$ & $65-69$ & $70-79$ & $\mathbf{8 0}$ e + & \\
\hline Ignorado/Branco & 28 & 22 & 16 & 8 & 74 \\
Sexual & 12 & 4 & 4 & - & 20 \\
Transfusional & 7 & - & 3 & - & 10 \\
Uso de Drogas Injetáveis & 1 & - & - & - & 1 \\
Hemodiálise & - & 1 & - & - & 1 \\
Domiciliar & - & 1 & 1 & - & 2 \\
Tratamento Cirúrgico & 2 & - & 1 & - & 3 \\
Tratamento Dentário & 2 & - & - & - & 2 \\
Outros & 9 & 1 & 2 & 1 & 13 \\
Total & 61 & 28 & 26 & 8 & 123 \\
\hline
\end{tabular}

Fonte: Ministério da Saúde - Sistema de Informação de Agravos de Notificação
Informações sobre a cobertura vacinal da população de idosos não estão disponibilizadas no SI-PNI do TABNET/ DATASUS. Os dados disponíveis referem-se à população geral do Estado, sem estratificação por faixa etária.

Assim, a cobertura vacinal de idosos residentes em Salvador foi analisada com base na proporção do número de doses aplicadas no período de 2004 a 2018, em relação à população vacinada. Nesse período, de acordo com os registros disponíveis no SI-PNI do SI-PNI do TABNET/DATASUS, 64.041 doses da vacina contra hepatite $B$ foram aplicadas em indivíduos com idade igual ou maior de 60 anos, em Salvador, o que corresponde a 19\% das 338.363 doses aplicadas no estado da Bahia, nesse mesmo período.

Na Tabela 3, é possível observar o número de doses da vacina aplicadas e a proporção de idosos vacinados contra hepatite B, no município de Salvador, no período de 2004 a 2018. Conforme pode ser verificado, em Salvador, nos anos 2017 e 2018, foram observados os maiores números de doses aplicadas (10.707 e 10.282, respectivamente). Nos anos anteriores, esses números são significativamente menores, especialmente em 2004. No período de 2004 a 2018, o maior número de doses aplicadas refere-se à primeira dose (27.442), seguida da segunda e da terceira doses (19.074 e 15.037, respectivamente). É importante ressaltar que a eficácia de 80 a $100 \%$ da vacina, para prevenção da infecção ou doença clínica causada pelo HBV, refere-se aos indivíduos que receberam o esquema vacinal completo, ou seja, as três doses ${ }^{20}$.

Os dados mostram aumento do número de doses aplicadas da vacina contra hepatite $B$, em idosos, no município de Salvador, especialmente nos anos 2017 e 2018, após a universalização da vacina em 2016. Todavia, é importante destacar que, no mesmo período, também houve aumento importante do número de indivíduos com idade igual ou acima de 60 anos, na população soteropolitana.

Tabela 3 - Proporção de idosos residentes em Salvador, Bahia, vacinados contra hepatite B, no período de 2004 a 2018.

\begin{tabular}{llllllllll}
\hline & & & \multicolumn{3}{c}{$\begin{array}{c}\text { Número (n) e proporção (\%) de idosos } \\
\text { Ano } \begin{array}{c}\text { População } \\
\text { estimada } \\
\text { de idosos }\end{array}\end{array}$} & \multicolumn{5}{c}{$\begin{array}{c}\text { vacinados contra hepatite B, por dose } \\
\text { da vacina }\end{array}$} \\
\cline { 3 - 9 } & 1a dose & 2a dose & 3a dose \\
\hline 2004 & 190.947 & 451 & 0,2 & 387 & 0,2 & 303 & 0,2 \\
2005 & 196.656 & 2.030 & 1,0 & 1.217 & 0,6 & 785 & 0,4 \\
2006 & 198.809 & 1.158 & 0,6 & 682 & 0,3 & 669 & 0,3 \\
2007 & 206.597 & 1046 & 0,5 & 718 & 0,3 & 634 & 0,3 \\
2008 & 226.796 & 832 & 0,4 & 388 & 0,2 & 688 & 0,3 \\
2009 & 236.855 & 1045 & 0,4 & 770 & 0,3 & 862 & 0,4 \\
2010 & 24.7646 & 1.182 & 0,5 & 871 & 0,4 & 790 & 0,3
\end{tabular}




$\begin{array}{rrrrrrrr}2011 & 250.914 & 831 & 0,3 & 599 & 0,2 & 427 & 0,2 \\ 2012 & 380.000 & 1.017 & 0,3 & 791 & 0,2 & 593 & 0,2 \\ 2013 & 376.000 & 1.756 & 0,5 & 1.175 & 0,3 & 804 & 0,2 \\ 2014 & 415.000 & 2.201 & 0,5 & 1.477 & 0,4 & 1.079 & 0,3 \\ 2015 & 446.000 & 2.311 & 0,5 & 1.441 & 0,3 & 1.170 & 0,3 \\ 2016 & 520.000 & 2.756 & 0,5 & 1.790 & 0,3 & 838 & 0,2 \\ 2017 & 540.000 & 4.716 & 0,9 & 3.478 & 0,6 & 2.513 & 0,5 \\ 2018 & 526.000 & 4.110 & 0,8 & 3.290 & 0,6 & 2.882 & 0,5 \\ \text { Total } & 4.958 .220 & 27.442 & 7,9 & 19.074 & 5,2 & 15.037 & 4,6\end{array}$

Fonte: Instituto Brasileiro de Geografia e Estatística/SI-PNI.

De acordo com os dados da tabela, as maiores proporções de idosos vacinados foram observadas nos anos de 2017 e 2018. Entretanto, nesse mesmo período, também foi observado crescimento significativo da população idosa, no município de Salvador. Dessa forma, os valores estimados das doses aplicadas em 2017 e 2018 correspondem, respectivamente, a 0,9\% e 0,8\% da população de indivíduos com idade igual ou superior a 60 anos residentes em Salvador. A análise das doses aplicadas, no período de 2004 a 2018, na população total estimada de 4.958.220 idosos, mostra que $27.442(7,9 \%)$ receberam a primeira dose, $19.074(5,2 \%)$ a segunda dose e 15.037 (4,6\%) a terceira dose da vacina. Esses dados sugerem que, além de baixa cobertura vacinal, há elevada prevalência de indivíduos com esquema vacinal incompleto contra hepatite $B$, na população idosa de Salvador.

\section{DISCUSSÃO}

Segundo relatório do Ministério da Saúde divulgado em 2018, apenas 17,25\% dos idosos brasileiros completaram o esquema vacinal contra a hepatite $B$, no período de 1994 a $2017^{11}$. A cobertura vacinal contra a doença, considerada adequada pelo $\mathrm{PNI} / \mathrm{MS}$, é de $95 \%$ na população ${ }^{9}$. O SI-PNI/DATASUS não fornece dados da cobertura vacinal em idosos, o que dificulta o acompanhamento dessa população. Estudos que evidenciem a situação vacinal contra hepatite $B$ na população idosa residente no município de Salvador, Bahia, são escassos. Pesquisa desenvolvida por Martins e Costa ${ }^{15}$, em 2015, revelou que a população total soteropolitana estava em situação de vulnerabilidade, com baixo número de indivíduos vacinados contra o HBV. Assim, as informações obtidas no presente estudo podem ser úteis para o planejamento e implementação de medidas que visem aumentar o número de idosos residentes em Salvador, com esquema de vacinação completo contra hepatite B.

Os dados obtidos revelaram que o número de idosos vacinados contra hepatite $B$, residentes em Salvador, em 2004 , foi o mais baixo no período investigado. Esta baixa taxa de adesão pode ser explicada pelo fato da vacina não ter sido incluída no calendário de vacinação do adulto e do idoso, instituído em abril do referido ano. Nos anos posteriores, apesar de apresentar uma maior proporção de idosos vacinados, só foi observado aumento significativo nos anos de 2017 e 2018, após a universalização da vacina em 2016. Entretanto, no período de 2004 a 2018, apenas $24,4 \%$ da vacina corresponderam à terceira dose, o que sugere baixa prevalência de idosos com esquema vacinal completo. Dessa forma, é muito relevante a formulação de estratégias de incentivo à ampliação da taxa de adesão de idosos à vacinação contra hepatite $B$.

Entre os fatores que podem influenciar a baixa adesão à vacina estão: hesitação vacinal; preconceito; insegurança; falta de conhecimento; e medo em relação aos efeitos adversos da vacina ${ }^{16}$. Mais recentemente, a divulgação de fake news é outro fator que tem recebido destaque por influenciar negativamente a adesão às vacinas. Segundo pesquisa realizada pela Sociedade Brasileira de Imunizações, com mais de 2 mil pessoas nas cinco regiões do país, 7 em cada 10 brasileiros acreditam em fakes news relacionadas a vacinas ${ }^{17}$.

Vários estudos foram realizados para investigar a prevalência da hepatite $B$, em diferentes grupos populacionais, mas poucos tiveram como objetivo analisar, especialmente, a prevalência dessa doença em indivíduos com idade igual ou superior a 60 anos. Em 2012, Pimentel, Schinioni e Freire $^{18}$ identificaram que, no período de 2007 a 2012, dentre os municípios do Estado da Bahia, com número acima de 100 mil habitantes, Salvador foi o de maior registro de casos de hepatite $B$, levando em consideração todas as faixas etárias. Além da elevada prevalência da enfermidade, Salvador se destaca, também, pelo envelhecimento crescente de sua população, indicada em projeções divulgadas pelo IBGE ${ }^{14}$. A maior longevidade da população requer a implantação de políticas públicas que visem prevenir doenças transmissíveis e promover melhor qualidade de vida aos idosos. A vacinação de idosos tem papel importante nesse processo, pois, além de contribuir para a promoção de saúde dessa população, tem impacto socioeconômico significativo na saúde pública decorrente da menor prevalência de agravos que acometem os idosos ${ }^{19}$.

É preciso ressaltar que dentre as hepatites virais, a hepatite $B$ é a segunda mais prevalente no país e que, por ser uma doença imunoprevenível, a vacina é ferramenta fundamental para redução de sua ocorrência nesse grupo populacional ${ }^{2}$.

Os avanços no tratamento da disfunção erétil e as terapias hormonais têm sido apontados como importantes fatores que contribuíram para o prolongamento da vida sexual ativa de indivíduos com idade igual ou superior a 60 anos. Contudo, esses avanços estão associados a maior resistência ao uso de preservativos por parte desse grupo populacional, o que tem contribuído para sua maior vulnerabilidade às doenças sexualmente transmissíveis, principalmente hepatite $\mathrm{B}^{5}$.

A prevenção da infecção pelo HBV é ainda mais importante para os idosos porque, nesse grupo, há maior risco de evolução para as formas graves da doença, incluindo 
cirrose hepática e carcinoma hepatocelular ${ }^{3}$.

Os dados obtidos neste estudo mostram que, apesar de a vacina contra hepatite $B$ estar incluída no Calendário de Vacinação do Idoso e estar disponível gratuitamente na rede pública de saúde desde 2016, a proporção de idosos vacinados no município de Salvador, Bahia, é baixa e a maioria dos indivíduos não completou o esquema vacinal de três doses. $\mathrm{O}$ aumento do número de doses aplicadas da vacina nos últimos dois anos, após a sua universalização, não foi proporcional ao crescimento contínuo da população idosa que, segundo projeção do IBGE, deve crescer ainda mais nas próximas décadas.

\section{CONCLUSÃO}

O presente estudo revelou que, de acordo com o Sistema de Informações do Programa Nacional de Imunizações (SI-PNI) do TABNET/DATASUS, a proporção de idosos vacinados com o esquema vacinal completo da hepatite $B$ no município de Salvador, Bahia, no período de 2004 a 2018, está abaixo do considerado adequado pelo Ministério da Saúde. A baixa cobertura vacinal, associada ao crescente envelhecimento da população soteropolitana, ressalta a importância da implementação de novas estratégias no planejamento de programas de atenção à saúde do idoso, com o objetivo de conscientizá-los a respeito da importância da vacinação completa contra a hepatite $B$.

\section{REFERÊNCIAS}

1 SERRA, J. Lista nacional de doenças de notificação compulsória. Inf. Epidemiol. SUS, Brasília, v. 9, n. 1, p. 59-60, mar. 2000. Disponível em: http://scielo.iec.gov.br/scielo.php?script=sci_arttext\&pi $d=$ S0104-16732000000100006. Acesso em: 5 nov. 2019.

2 BRASIL. Ministério da Saúde. Secretaria de Vigilância em Saúde. Departamento de Vigilância, Prevenção e Controle das IST, do HIV/Aids e das Hepatites Virais. Boletim Epidemiológico - Hepatites Virais 2018. Brasília, DF: Ministério da Saúde, 2019.

3. HOAN, X.N. et al. Vitamin D deficiency and hepatitis viruses-associated liver diseases: A literature review. World J. Gastroenterol., v. 24, n. 4, p. 445-460, Jan 2018. Acesso em: 10 jun. 2019.

4. IBGE. Pesquisa Nacional por Amostra de Domicílios Contínua. Número de idosos cresce 18\% em 5 anos e ultrapassa 30 milhões em 2017. Agência IBGE de Notícias, Brasília, DF, 2018. Disponível em: https:// agenciadenoticias.ibge.gov.br/agencia-noticias/2012-agencia-de-noticias/ noticias s/20980-numero-de-idosos-cresce-18-em-5-anos-e-ultrapassa30-milhoes-em-2017. Acesso: 10 jun. 2019.

5. DORNELAS NETO, J. et al. Doenças sexualmente transmissíveis em idosos: uma revisão sistemática. Ciênc. saúde coletiva, Rio de Janeiro, v. 20, n. 12, p. 3853-3864, 2015. Acesso em: 10 jun. 2019.

6. BRASIL. Ministério da Saúde. Secretaria de Vigilância em Saúde. Departamento de Vigilância Epidemiológica. Hepatites virais: o Brasil está atento. Brasília, DF: Ministério da Saúde, 2008. Acesso em: 05 nov. 2019.

7. TARCHA, N. I. Prevalência e fatores associados à infecção pelo vírus da hepatite B entre idosos do município de São Paulo, Brasil: estudo SABE. 2018. Dissertação (Mestrado em Ciências) - Faculdade de Saúde Pública, Universidade de São Paulo, São Paulo, 2018. p. 17-18. Disponível em: https://teses.usp.br/teses/disponiveis/6/6143/tde-03102018-082714/
publico/NoemilannoneTarcha_MTR_ORIGINAL.pdf. Acesso em: 05 nov. 2019.

8. FERREIRA C. T.; SILVEIRA, T.R. Hepatites virais: aspectos da epidemiologia e da prevenção. Rev. Bras. Epidemiol., São Paulo, v. 7, n.4, p. 473-487, 2004. Acesso em: 01 nov. 2019.

9. BRASIL. Ministério da Saúde. Secretaria de Vigilância em Saúde. Programa Nacional de Imunizações: aspectos históricos dos calendários de vacinação e avanços dos indicadores de coberturas vacinais no período de 1980 a 2013. Boletim Epidemiológico, Brasília, v. 46, n. 30, p. 2-13, 2015. Disponível em: http://portalarquivos2.saude.gov.br/images/pdf/2015/ outubro/14/besvs-pni-v46-n30.pdf. Acesso em: 01 nov. 2019.

10. BRASIL. Ministério da Saúde. Nota Informativa $n^{\circ}$ 149, de 2015/ CGPNI/DEVIT/SVS/MS. Informa as mudanças no Calendário Nacional de Vacinação para o ano 2016. Brasília, DF: Ministério da Saúde, 2015. Disponível em: http://www.aids.gov.br/pt-br/legislacao/nota-informativano-1492015. Acesso em: 01 nov. 2019

11. BRASIL. Ministério da Saúde. Secretaria de Vigilância em Saúde. Departamento de Vigilância das Doenças Transmissíveis. Coordenação Geral do Programa Nacional de Imunizações. Quedas nos índices de cobertura vacinal no Brasil ano de 2018. Brasília, DF: Ministério da Saúde, 2019. Disponível em: https://portal.cfm.org.br/images/PDF/2018_encm_ magdarodrigues.pdf. Acesso: 10 out. 2019.

12. BRASIL. Ministério da Saúde. DATASUS. Sistema de Informações do Programa Nacional de Imunizações (SI-PNI). Imunizações doses aplicadas -Brasil. Brasília, DF: Ministério da Saúde, 2018? Disponível em: http:// tabnet.datasus.gov.br/cgi/dhdat.exe?bd_pni/dpnibr.def. Acesso em:29 out. 2019.

13. BRASIL. Ministério da Saúde. DATASUS Sistema de Informação de Agravos de Notificação de (SINAN). Hepatites virais - Casos confirmados notificados no Sistema de Informação de Agravos de Notificação-Brasil. Brasília, DF: DATASUS, 2018? Disponível em: http://tabnet.datasus.gov. br/cgi/deftohtm.exe?sinannet/cnv/hepabr.def. Acesso em: 29 out. 2019.

14. INSTITUTO BRASILEIRO DE GEOGRAFIA E ESTATÍSTICA (IBGE). Sistema IBGE de Recuperação automática-SIDRA. Brasília, DF: 2019? Disponível em: https://sidra.ibge.gov.br/tabela/6407. Acesso em: 01 nov. 2019

15. MARTINS, M.M.F.; COSTA, E.A.M.A. Aspectos epidemiológicos e estado vacinal para hepatite $B$ no município de Salvador, Bahia. Rev. Ciênc. Méd. Biol., Salvador, v. 14, n.2, p. 160-164, 2015. Acesso em: 01 nov. 2019

16. SUCCI, R. C. M. Recusa vacinal - que é preciso saber. J. Pediatr., Porto Alegre, v. 94, n. 6, p. 574-581, 2018. Acesso em: 01 nov. 2019.

17. SOCIEDADE BRASILEIRA DE IMUNIZAÇÕES. As fake news estão nos deixando doentes? São Paulo: 2019. Disponível em: https://sbim.org. $\mathrm{br} /$ acoes/as-fake-news-estao-nos-deixando-doentes. Acesso em 20 nov. 2019.

18. PIMENTEL, R.; SCHINIONI, M.I.; FREIRE, S. M. Aspectos epidemiológicos da hepatite $B$ a partir do Sistema de Informação de Agravos de nitrificação (SINAN) do Estado da Bahia. Rev. Ciênc.Méd. Biol. v. 11, n. 2, p. 207-211, 2012. Acesso em: 10 nov. 2019.

19. SOCIEDADE BRASILEIRA DE IMUNIZAÇÕES. Imunização de adultos e idosos: bases para estudos e decisões 2019. São Paulo: 2019. Disponível em: https://sbim.org.br/images/books/forum-imunizacao-de-adultosidosos-2019.pdf. Acesso em: 01 jul. 2019.

20. SOCIEDADE BRASILEIRA DE PEDIATRIA. Vacina contra hepatite B. Rev. Assoc. Med. Bras., São Paulo, v. 52, n. 5, p. 288-289, 2006.

Submetido em: 04/11/2019

Aceito em: 08/01/2020 